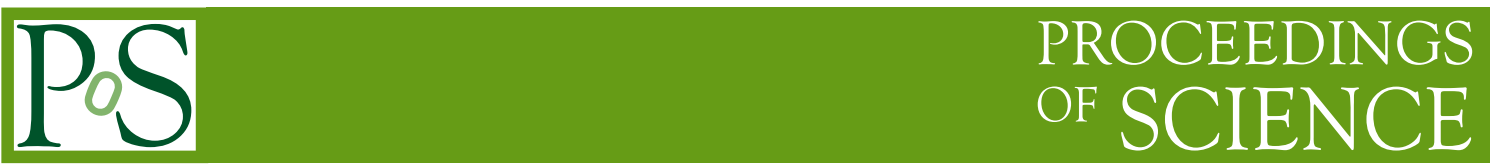

\title{
Estimation of the material budget of the CMS Tracker using the triplet method
}

\author{
Suvankar Roy Chowdhury for the CMS Collaboration ${ }^{a, *}$ \\ ${ }^{a}$ Scuola Normale Superiore di Pisa, \\ Piazza dei Cavalieri, 7, Pisa, Italy \\ E-mail: sroychow@cern.ch
}

Silicon trackers are used extensively in high energy physics experiments. Estimation of the material budget of the tracker is crucial for the physics program of any experiment, especially for precision measurements, as it has a direct impact on reconstruction performance and calculation of systematic errors. In this paper, a novel method of estimating the material budget of the CMS Tracker using three consecutive hits from a reconstructed track is presented. Comparisons are made between the material budget obtained from data, and simulated events.

40th International Conference on High Energy physics - ICHEP2020

July 28 - August 6, 2020

Prague, Czech Republic (virtual meeting)

\footnotetext{
${ }^{*}$ Speaker
} 


\section{Introduction}

A common feature of tracking systems in modern collider based experiments, e.g. in the ATLAS [1] and CMS [2] experiments at the Large Hadron Collider (LHC), and the Belle II [3] experiment at $\mathrm{KeK}$, is that they have multiple layers of silicon detectors. As charged particles pass through the silicon sensors, ionization gives rise to a signal in individual channels of each detector. Hits in multiple layers of a tracking system are then used to reconstruct charged particle tracks. One consideration in the design of these systems is the overall minimisation of material in the tracking volume, also known as the material budget, in order to reduce eventual impact on reconstruction and physics performance. In this paper, a novel method to precisely determine the material budget of the CMS tracker [4] is presented. The idea is to form a small track segment, known as a triplet, built from hits in three consecutive layers of the tracker and use the local sagitta of this triplet to estimate the material in the middle layer. Applying this technique to the original configuration of the CMS pixel detector [4], a comparison will be made between simulated events and Run 2 collision data, before the pixel detector was upgraded in 2017. The coordinate system of the CMS experiment is explained in detail in [2]. The azimuthal angle $(\phi)$ is measured from the $x$-axis in the $\mathrm{x}-\mathrm{y}$ plane, whereas the polar angle $(\theta)$ is measured from the z-axis. Pseudorapidity is defined as, $\eta=-\ln \tan (\theta / 2)$.

\section{Triplet method}

Let us consider a system of three consecutive hits along a track, referred to as a triplet. The local trajectory of the triplet deviates from a straight line because charged particles bend in the magnetic field and because they also undergo multiple Coulomb scattering. The latter is the dominant effect for low momentum tracks. In our model, we assume that for a given triplet, multiple scattering takes place only in the central layer of the triplet. The hits can be transformed to the global frame and represented in the transverse $(\mathrm{r}-\phi)$ plane using a set of $2 \mathrm{D}$ vectors.
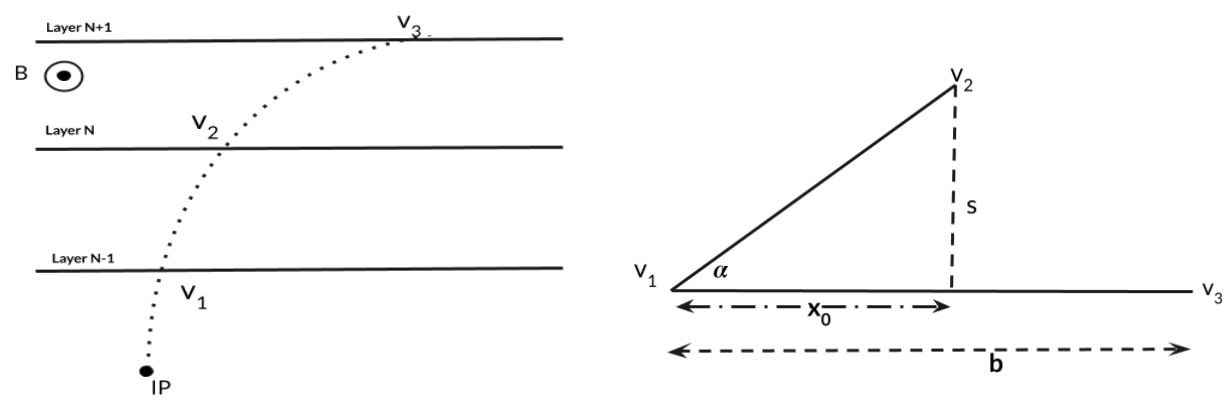

Figure 1: Left: representation of a track crossing three consecutive layers with hit positions at $v_{1}, v_{2}$ and $v_{3}$ projected onto the transverse place. Right: local geometry of the system of three consecutive hits [5]. Multiple Coulomb scattering happens at $v_{2}$ and $\mathrm{s}$ is the sagitta of the local trajectory of the triplet.

Such a system of triplets is shown in Fig.1 (left), where $v_{1}, v_{2}$ and $v_{3}$ are the measured points of the three layers in order of increasing radius. We define a system of coordinates, shown in Fig.1 (right), with $v_{1}$ as the origin, the $x$-axis as the line joining $v_{1}$ and $v_{3}$, and the $y$-axis orthogonal 
to the $x$-axis. Assuming multiple scattering takes place in the middle layer, at $x_{0}=a \cos \alpha$, with an angle $\Theta$, the local trajectory of a track projected on the transverse plane in this system of coordinates can be calculated, using the formalism described in [5]. The local sagitta $s$ can be expressed as,

$$
\frac{s}{x_{0}\left(b-x_{0}\right)}=-\frac{1}{2 \rho}-\frac{\Theta}{b}
$$

where $\rho$ is the radius of curvature of the track. In this model, as stated earlier, the assumption is that multiple scattering takes place only in the middle layer. However, (1) is also a good approximation of the local trajectory with an effective angle $\Theta$, which encodes the multiple scattering angles weighted with some geometrical function as a more general case. This is because the closer the material to the first or the last layer, the smaller the contribution to the effective scattering angle $\Theta$. Multiplying (1) by the transverse momentum $p_{\mathrm{T}}$ of the track, measured from a global fit across several layers, we get,

$$
t \equiv \frac{p_{\mathrm{T}} s}{x_{0}\left(b-x_{0}\right)}=-\frac{p_{\mathrm{T}}}{2 \rho}-\frac{p_{\mathrm{T}} \Theta}{b} .
$$

The quantity $t$ can be measured on an event by event basis from the three projected coordinates $\vec{v}_{1}, \vec{v}_{2}, \vec{v}_{3}$ and the global $p_{\mathrm{T}}$ of the track. Since the multiple scattering angle $\Theta$ has an expectation value of zero [6], the expectation value of $t$ is $\frac{p_{\mathrm{T}}}{2 \rho}$, i.e. the ratio between the transverse momentum and the local radius of curvature, which is constant in magnitude for a given triplet and changes sign depending on the charge of the track. The $p_{\mathrm{T}}$ and the radius of curvature $(\rho)$ of a track are linked to the magnetic field $(B)$ as, $p_{\mathrm{T}}= \pm \frac{0.3 B \rho}{100}$, where $p_{\mathrm{T}}$ is in $\mathrm{GeV}, \rho$ is measured in $\mathrm{cm}$ and $B$ is measured in Tesla. Assuming a uniform magnetic field of $3.8 \mathrm{~T}$ corresponding to that of the CMS solenoid one gets, $\frac{p_{\mathrm{T}}}{2 \rho}= \pm 5.7 \times 10^{-3} \mathrm{GeV} / \mathrm{cm}$. Figure 2 shows a plot of the quantity $t$ obtained with hits measured by the three barrel pixel layers of the CMS detector. The two gaussian like peaks are produced by particles with positive and negative charges respectively. The spread of the

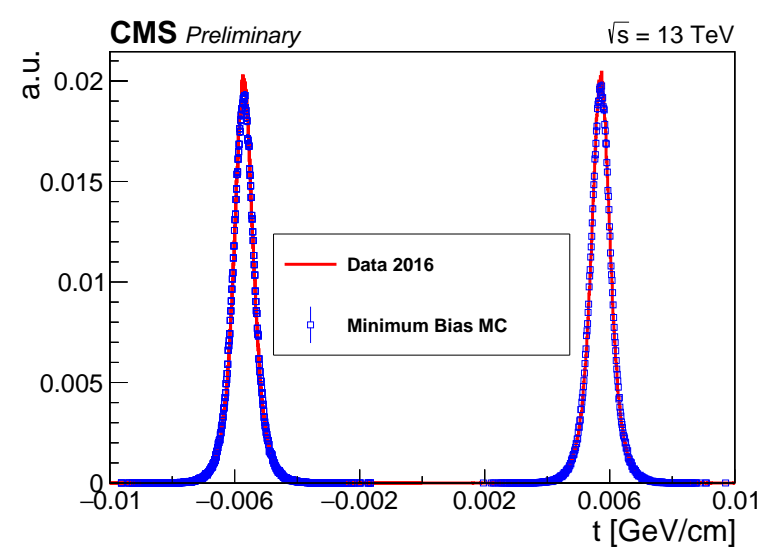

Figure 2: Distributions of the variable $t$ for triplets defined by hits in the three barrel layers of the CMS pixel detector situated at mean radii of 4.4,7.3 and $10.2 \mathrm{~cm}$ from the beam line.

distribution $t$ includes the finite resolution from the measurements of $p_{\mathrm{T}}$ and the hit positions $\vec{v}_{i}$, as well as the effect of multiple scattering as described in the last term of (2). The spread of the 
multiple scattering angle $\Theta$ is given by [7],

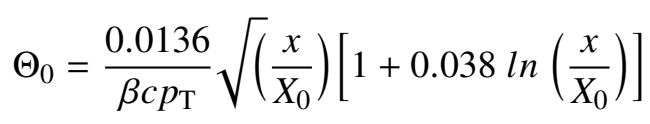

where $\beta c$ is the velocity of the charged particle, and $\frac{x}{X_{0}}$ is the thickness of the scattering layer in radiation lengths.

The quantitites $p_{\mathrm{T}}, s, x_{0}$ and $\left(b-x_{0}\right)$ are all measured with finite precision and contribute to the error on $t$. Propagating the uncertainties using (2), we obtain,

$$
\Delta t=t_{0} \frac{\Delta p_{\mathrm{T}}}{p_{\mathrm{T}}} \oplus t_{0} \frac{\Delta x_{0}\left(b-x_{0}\right)}{x_{0}\left(b-x_{0}\right)} \oplus \frac{p_{\mathrm{T}} \Delta s}{x_{0}\left(b-x_{0}\right)} \oplus \Delta\left(\frac{p_{\mathrm{T}}}{b} \Theta\right)
$$

where $t_{0}$ is average value of $t$ computed for tracks of a given charge. Using typical values $\frac{\Delta p_{\mathrm{T}}}{p_{\mathrm{T}}} \approx 1 \%$, $\Delta x_{0} \simeq \Delta\left(b-x_{0}\right) \approx 10 \mu \mathrm{m}, x_{0} \simeq\left(b-x_{0}\right) \approx 10 \mathrm{~cm}$ and $\Delta s \approx 10 \mu \mathrm{m}$, the terms in (4) can be derived. Table 1 summarises their values.

Table 1: Estimates of the four terms on the right hand side of (4). All numbers are in $\mathrm{GeV} / \mathrm{cm}$.

\begin{tabular}{|c|c|c|c|}
\hline$t_{0} \frac{\Delta p_{\mathrm{T}}}{p_{\mathrm{T}}}$ & $t_{0} \frac{\Delta x_{0}\left(b-x_{0}\right)}{x_{0}\left(b-x_{0}\right)}$ & $\frac{p_{\mathrm{T}} \Delta s}{x_{0}\left(b-x_{0}\right)}$ & $\Delta\left(\frac{p_{\mathrm{T}}}{b} \Theta\right)$ \\
\hline $6 \times 10^{-5}$ & $1 \times 10^{-6}$ & $\frac{p_{\mathrm{T}}}{1 \mathrm{GeV}} \frac{\Delta s}{10 \mu \mathrm{m}} 10^{-4}$ & $\sqrt{\frac{x / X_{0}}{0.01}} 10^{-4}$ \\
\hline
\end{tabular}

The last two terms in (4) are the major contributors to the variance of the distribution of $t$ as shown in Table 1. In order to separate the contribution of the sagitta uncertainty (third term) from the one of the multiple scattering contribution (fourth term), a study of the variance of the peaks as a function of $p_{\mathrm{T}}^{2}$ would indicate whether the uncertainty on the sagitta is dependent on the transverse momentum itself. A linear relationship would prove that the error on the sagitta is independent of the momentum. This will be demonstrated in the next section. At $p_{\mathrm{T}}=0$, the only term contributing to $\Delta t$ comes from the last term of (4) which can be approximated using (3) as,

$$
\operatorname{Var}\left(\frac{p_{\mathrm{T}} \Theta}{b}\right) \simeq \frac{x}{X_{0}}\left(\frac{0.0136}{b}\right)^{2} \mathrm{GeV}^{2} / \mathrm{cm}^{2}
$$

\section{Validation with CMS data}

A validation of the model was performed using Run 2 data collected by the CMS experiment in 2016. The events from data were collected with a Zero Bias trigger where the detector is read out randomly during p-p collisions. These events are mostly dominated by soft QCD interactions with predominantly hadronic final state content, distributed uniformly over pseudorapidity $\eta$, and with an exponentially decreasing $p_{\mathrm{T}}$ spectrum. To compare the accuracy of the material distribution in simulation, simulated events with the same characteristics as the Zero Bias data were used. For the analysis, tracks were selected with the following criteria:

- Transverse momentum between $750 \mathrm{MeV}$ and $1.5 \mathrm{GeV}$. The lower threshold is to reject tracks which do not traverse the entire radial length of the tracker. The upper cut is arbitrary but is large enough to obtain a significant range in $p_{\mathrm{T}}^{2}$. 
- The fully reconstructed track should have at least 14 hits.

- The relative uncertainty on the transverse momentum measurement should be less than $1 \%$.

Since the material budget has strong dependence on $\eta$, the sample of selected tracks is divided into 25 bins of $\eta$ for the position of the hit in the middle layer of the triplet. Here we consider triplets formed by hits in the first, second and third barrel layers of the pixel detector (BPix), corresponding to points $v_{1}, v_{2}$ and $v_{3}$ of the triplet system shown in Fig. 1. For those tracks the quantity $t$ defined in (2) and integrated over all $\eta$ bins and all track transverse momenta is shown in Fig. 2.

Following (2) we compute the variance of the quantity $\left(t-t_{\text {mean }}\right) b$ for positive (negative) tracks, where $t_{\text {mean }}$ is the mean of the quantity $t$ computed for positive (negative) tracks. Since the two peaks have equal numbers of events, we take the average of the two variances. This is done for each of the 25 bins in $\eta$ and for each $\eta$ bin in ten bins of $p_{\mathrm{T}}$. Figure 3 shows the measured variances in data (left) and simulation (right) plotted as a function of $p_{\mathrm{T}}^{2}$ for $0<\eta<0.2$. From Section 2 and using (5), a straight line fit to this distribution gives an intercept at zero which is proportional to the material thickness in radiation lengths in the middle layer of the triplet.
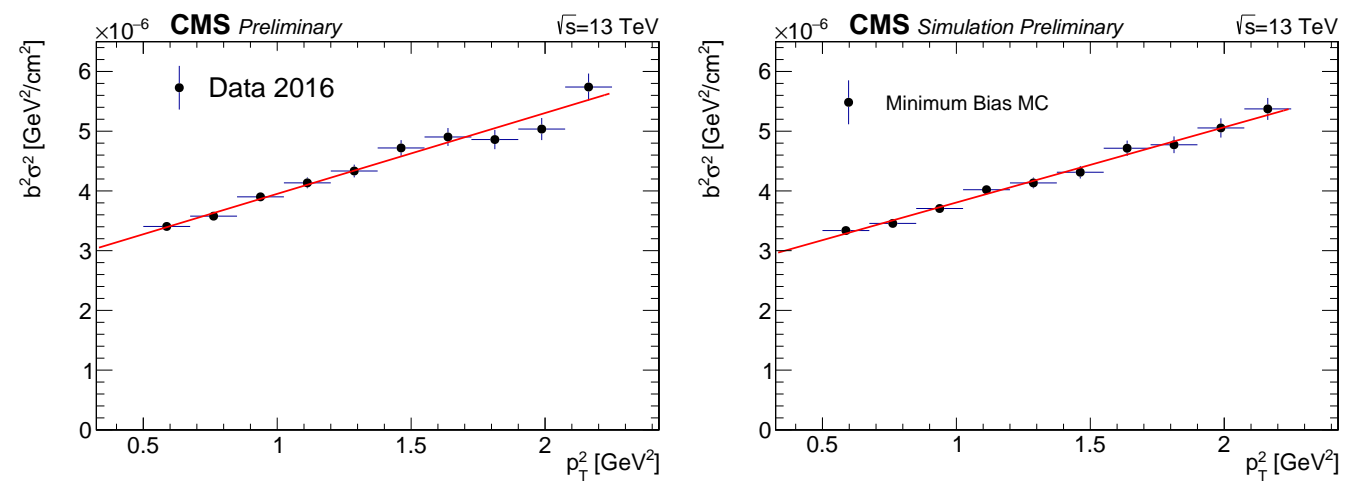

Figure 3: Fit of the variance of the peaks of the $t$ distribution as function of $p_{\mathrm{T}}^{2}(0<\eta<0.2)$ for data (left) and simulation (right).

A plot of the material thickness in radiation lengths as a function of $\eta$ for BPix layer 2 is shown in Fig. 4 (left). The figure describes the increase in material traversed by particles as they impact the layer at oblique angles, which is typical for a barrel detector of constant thickness. The minimum is at $\eta \approx 0$ and it increases symmetrically.

The assumption that the variance of the distribution of $t$ is dominated by the two last terms of (4) can be checked by multiplying the measured variance by $\sin (\theta)$ of the track. This is shown in Fig. 4 (right). The fact that this plot is flat shows that the constant contribution to the variance of the first two terms of (4) is indeed small. For the two bins at the edges, the fluctuations in the computed radiation length are due to the fact that the assumption of constant material no longer holds true in this region (increased material from service cables, cooling pipes etc). For the central regions in pseudorapidity however, there is a good correspondence between estimates extracted from both simulation and collision data samples, for both distributions in Fig. 4. 

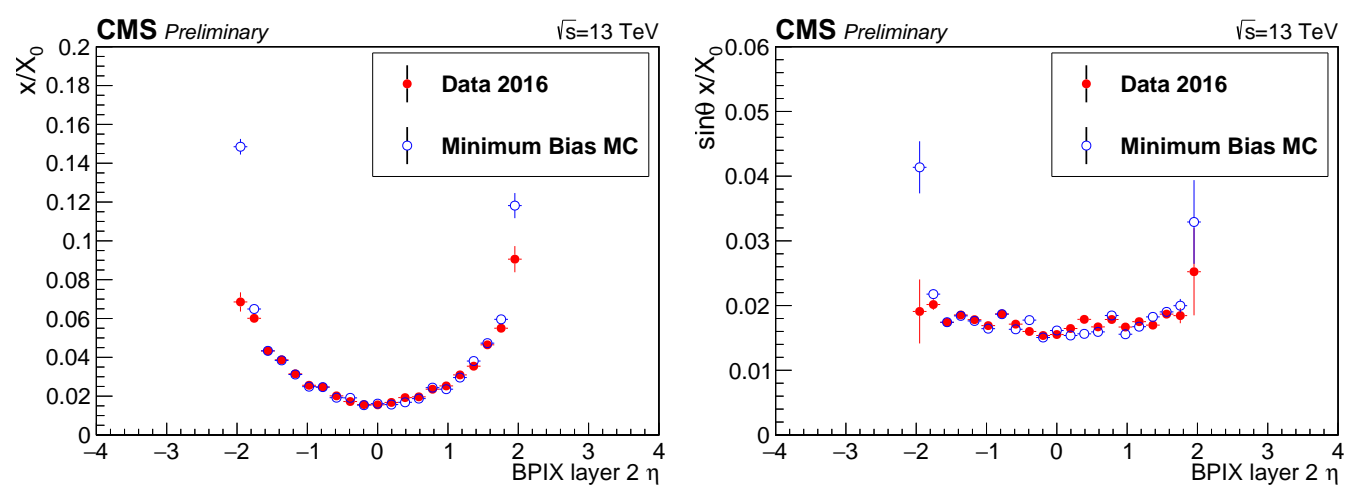

Figure 4: Left: distribution of estimated material thickness in radiation lengths $\left(x / X_{0}\right)$ for triplets reconstructed from both data and simulation, as a function of $\eta$ for the hit in BPix layer 2. Right: distribution of $\sin (\theta) x / X_{0}$, where $\theta$ is the polar angle of the track, for the same system of triplets.

\section{Conclusion}

A new method of estimating the material budget of the CMS tracker in situ, with $\sim 1 \mathrm{GeV}$ tracks, has been presented. It assumes that multiple scattering happens in the central layer of the triplet. The material budget has been measured in data and simulation for the pixel detector of CMS and good agreement is seen. Further studies are ongoing to validate the method with the CMS strip tracker.

\section{References}

[1] ATLAS Collaboration, "The ATLAS Experiment at the CERN Large Hadron Collider", JINST 3 (2008) S08003. doi:10.1088/1748-0221/3/08/S08003

[2] CMS Collaboration, "The CMS experiment at the CERN LHC", JINST 3 (2008), S08004. doi:10.1088/1748-0221/3/08/S08004

[3] Belle-II Collaboration, "Belle II Technical Design Report", KEK-REPORT-2010-1 (2010). arXiv: 1011.0352

[4] CMS Collaboration, "The CMS Tracker system project: Technical Design Report", CERN LHCC/98-6, CMS-TDR-5 (1998).

[5] E. Manca, "Validation of the muon momentum resolution in view of the W mass measurement with the CMS experiment", CERN-THESIS-2016-173(2016). https://cds.cern.ch/record/2233647

[6] J.B. Marion and B.A. Zimmerman, "Multiple scattering of charged particles", Nuclear Instruments and Methods, 51 193-101 (1967).

[7] P.A. Zyla et al., "Particle Data Group", Prog. Theor. Exp. Phys. 2020, 083 C01 (2020). 